\title{
La salud como un derecho y la atención de los pacientes no COVID-19
}

\author{
The right to health and care of non-COVID-19 patients
}

\author{
Percy Díaz -Morón ${ }^{1, a}$
}

\section{Sr. Editor:}

El derecho a la salud es una condición innata que tiene la persona y es protegido por el Estado quien debe adjudicar recursos y diseñar políticas públicas para garantizarlo, este no solo abarca la atención de salud oportuna y apropiada, sino también los principales determinantes de la salud, como el acceso al agua potable, condiciones sanitarias adecuadas, alimentos sanos, vivienda adecuada, y condiciones sanas en el trabajo y en el medio ambiente.

En la actualidad, nuestro país enfrenta una pandemia a causa de la nueva enfermedad por el coronavirus (COVID-19) que pone a prueba al Gobierno en su sistema de salud, poniendo en evidencia su precariedad y dificultades técnico-normativas, que conlleva a una inadecuada operatividad y total descuido de este, persistiendo la falta de acceso de la población a servicios sanitarios de calidad a pesar de la política de aseguramiento universal implementada.

El Aseguramiento Universal en Salud si bien es cierto plantea cambios institucionales en el sistema de salud para garantizar el derecho pleno y progresivo de la población a la seguridad social, pero según la Constitución, en la práctica, no se cumple este derecho, pues existe un abandono del primer nivel de atención y ausencia de políticas adecuadas de gestión hospitalaria, situación que se manifiesta en la presencia de nosocomios sin la cantidad suficiente de médicos especialistas, infraestructura inadecuada, falta de equipamiento, inadecuado abastecimiento de medicamentos e insumos, y escasa articulación multisectorial para abordar el problema sanitario.

Se ha creado una barrera de acceso a la población con necesidades de salud y problemas de emergencias y urgencias No COVID-19. (Enfermedades crónicas degenerativas, infecciosas, oncológicas, hematológicas, pediátricas, neuroquirúrgicas, etc.) como consecuencia de la crisis sanitaria por el COVID-19, por lo que se requiere implementar intervenciones sanitarias.

Se necesita, entonces, un sistema de salud bien articulado que permita atender a los pacientes COVID-19 y a las personas con otras necesidades de salud y problemas de emergencias y urgencias no COVID-19. Para ello se debe reorientar las estrategias actuales considerando priorizar las intervenciones sanitarias y garantizar la disponibilidad de recursos de salud para su atención, mediante un flujo diferenciado de organización para la atención, además de mejorar los mecanismos de coordinación y articulación interinstitucional e interinstitucional de todos los subsistemas de salud para garantizar la oportunidad de atención que contribuya a disminuir la morbimortalidad por todas sus causas.

\footnotetext{
1. Universidad Señor de Sipán. Escuela de Medicina Humana, Hospital Regional Lambayeque. Lambayeque, Perú.

a. Magíster en Ciencias. Especialista en Medicina Familiar y Comunidad.
} 
El escenario actual es una oportunidad para desarrollar la telemedicina, la cual el Ministerio de Salud ha venido implementando de forma lenta, fragmentada, restringida y de manera equivocada para el diagnóstico y tratamiento de pacientes, a pesar de ser validada por la Organización Mundial de la Salud y empleada en el mundo. Las recientes directivas del Ministerio de Salud, tampoco la consideraron, limitándola a consejería, tele orientación y asesoría en salud de personas con enfermedades crónicas, inmunizaciones y desarrollo infantil, tuberculosis, salud mental, VIH/SIDA y estado de salud de las gestantes ${ }^{2}$.

Se debe optar por operativizar la telemedicina en la red pública y privada de salud que incorpore a todos los establecimientos de salud (Minsa, Es Salud, FFAA, PNP, Privados, etc) regulando e implementando su formato de historia clínica electrónica en un escalamiento total e interconectado, esta medida con certeza reducirá el déficit de cobertura de atención médica, que supera más del $50 \%$ de la población que refiere algún problema de salud no Covid-19 en este escenario de pandemia. Personas con necesidades de salud de atención primaria y pacientes con patologías crónicas degenerativas, infecciosa, oncológica, que requieren continuidad de tratamiento, apoyo al diagnóstico y rehabilitación, que por las barreras de acceso generadas no son atendidos en los establecimientos de salud y hospitales.

Finalmente, para garantizar el derecho a la salud el objetivo del sistema de salud debe ser que cada peruano reciba servicios de salud de calidad, en cualquier centro de atención, público o privado, y pueda acceder de manera electrónica a su información clínica integrada; objetivo que puede lograse con un sólido respaldo político, compromiso intersectorial, estrategias claves del estado y la sociedad civil, que le asegure los recursos necesarios y legitimidad para su implementación y sostenibilidad en el tiempo, adecuado al proceso de descentralización y la implementación del Aseguramiento Universal en Salud.

Conflictos de interés: El autor declara que no existe conflicto de interés.

Fuentes de inanciamiento: Autofinanciado

\section{REFERENCIAS BIBLIOGRÁFICAS}

1. Díaz Javier; Beverinotti J, Andrian G. Impacto del COVID-19 en las Economías de la Región Andina. BID. 2020 Abr. Disponible en: http://dx.doi. org/10.18235/0002281

2. Ministerio de Salud. Perú. Estadísticas. - 2020. disponible en: http://www. minsa. gob.pe.

3. RM No 365-2008/MINSA. Perú. Aprueba la NTS N067-MINSA/DGSP-V.01 "Norma Técnica de Salud en Telesalud" disponible en: http://www.minsa. gob.pe.

4. Cárdenas E, Juárez C, Moscoso J, Vivas J. Determinantes Sociales de la Salud. Serie Gerencia para el Desarrollo 61 ESAN. Edición no venal. 2017,
231 Págs. Disponible en: https://repositorio.esan.edu.pe/bitstream/ handle/20.500.12640/1215/SGD_61.pdf

5. Organización Mundial de la Salud. Objetivos del Desarrollo Sostenible al 2030. Washington, D.C: OMS, 2017. Disponible en: https://www.who. int/topics/sustainable-development-goals/targets/es/.

6. Colegio Médico del Perú. El Sistema de Salud en el Perú - Situaciones y Desafíos. Lima: CMP, 2016. Disponible en:

http://www.cmp.org.pe/wp-content/uploads/2016/12/libroSistemaSaludPeru-.pdf

7. Alva G. Los Problemas de Salud del Perú Cómo Curar una Enfermedad. Conexión ESAN. 2017 Disponible en: https://www.esan.edu.pe/ conexion/actualidad/2017

8. Ministerio de Salud. Plan Nacional Concertado de Salud 2007-2020. Lima, Perú: 2007. Disponible en: http://bvs.minsa.gob.pe/local/MINSA/000_PNCS.pdf

9. "Mejorando los resultados de salud mediante el fortalecimiento de los derechos de los usuarios y de la gestión del sector público", Informe $\mathrm{N}^{\circ}$ 59218 - PE Unidad de Gestión del Sector de Desarrollo Humano, Unidad de Gestión de Países Andinos, Región de América Latina y el Caribe (español). Washington, DC: Grupo del Banco Mundial; 2011. Disponible en: http://documents.worldbank.org/curated/en/327961468057377453/ Peru-Recurso-Programatico-AAA-fase-IV-mejorando-los-resultados-de-salud-mediante-el-fortalecimiento-de-los-derechos-de-los-usuarios-y-de-la-gestion-del-sector-publico

10. Arce, M. "Implementación del aseguramiento universal en salud en regiones piloto del Perú", en Revista Peruana de Medicina Experimental y Salud Pública. 2009. 